\title{
Internet of Things (IOT) based Smart Environment integrating various Business Applications
}

\author{
Vimal Jerald A. \\ Dept. of Computer Science, \\ St. Joseph's College, \\ Tiruchirappalli, Tamilnadu, India
}

\author{
Albert Rabara S. \\ Dept. of Computer Science, \\ St. Joseph's College, \\ Tiruchirappalli, Tamilnadu, India
}

\author{
Daisy Premila Bai T. \\ Dept. of Computer Science, \\ St. Joseph's College, \\ Tiruchirappalli, Tamilnadu, India
}

\begin{abstract}
Internet of Things (IoT) plays a vital role in Next Generation Networks. Ample number of research works in IoT is carried out in developing countries like India. Research and Development units of industries are working on connecting tiny devices and objects to infer and to measure environmental and ecological resources. Domestic applications are also in line with this research. This paper proposes an integrated smart environment based on IoT. Several sectors like agriculture, security and emergency, banking, Surveillances, meteorology, health care, education, government - e services, domestic appliances monitoring, traffic surveillance are integrated and the various objects and devices are connected using RFID technology. This paper also deals with how various sectors are connected by means of RFID technology and sensor networks. It also brings forth an idea of establishing IoT information Kendra which infers and processes the data extracted by the various sectors in the smart environment. Functioning of a cloud application based computing and data centre and an administration and management centre attached to IoT information Kendra is also discussed.
\end{abstract}

\section{Keywords}

Internet of Things (IoT), RFID, IoT Information Kendra, Smart Environment.

\section{INTRODUCTION}

Internet of Things [IoT] is a prevalent technology that is being emerged in information technology today. Since 1991 when IoT was proposed by Kevin Ashton, several research works are carried out in the field of IoT and its relevant technologies. Internet of (IoT) is an integrated part of Future Internet and could be defined as dynamic global network infrastructure with self configuring capabilities based on standard and interoperable communication protocols where physical and virtual 'things' have identities, physical attributes and virtual personalities and use intelligent interface and are seamlessly integrated into the information network [1]. Today, the term Internet of Things goes beyond Machine to Machine (M2M) communication by connecting of devices, systems and services that goes beyond covers variety of protocols, domains and applications. A survey by Gartner says there will be nearly 26 billion devices in IoT by 2020 [2]. The proliferation of many natural resources and devices which measure, infer and understand environmental indicators in communicating and actuating network which creates Internet of Things [3]. Earlier, all the physical objects are tagged and uniquely identified using RFID transponders and readers. Now, IoT has grown to the level of comprising various networks of applications, computers, devices and objects as well that are interconnected using mobile technologies like wired, wireless and mobile networks, Bluetooth, GPS systems and other evolving technologies [4].

The intelligent identification, positioning, tracking, monitoring and management system has been put into applications in various fields [5]. These days IoT has gained popularity by some of its applications like intelligent transport, electric meter reading, telemedicine monitoring and so on. Health care, transport, emergency services, defense, crowd monitoring, infrastructure monitoring, environment monitoring, building management and water quality check are fewer potential applications of IoT identified by different focus groups of Melbourne city [3]. Establishing a smart environment integrating various applications and domains may be feasible using IoT. Research works on the domain has its boundaries around a single domain or sector. IoT relying on exchange of information through radio frequency identification (RFID) and this has resulted various applications ranging from healthcare, construction, hospitality to transportation and many more [6]. The mentioned applications in various sectors have been used to develop a smart environment. In contrast, the proposed architecture is trying to establish a smart environment integrating different sectors and their applications using RFID technology and sensor network. IoT infrastructure requires integration of several complimentary technologies like sensor networks, RFID system, mobile communication, conventional network and desktop environment [7].

IoT consists of objects, sensor devices communication infrastructure, computational and processing unit that may be placed on cloud, decision making and action invoking system [8] IoT things and devices play a vital role in business, information and social processes where they are enabled to interact and communicate among themselves and with the environment by data exchange about environment. They also react real and physical world events and influencing by running processes that trigger different actions and services with or without human intervention [1]. In the proposed work, a new concept of IoT information Kendra is introduced for processing data inferred from different sectors using RFID and sensor networks. The IoT information Kendra is complimented with cloud based data and service centre and an administrative management centre. A layered architecture consists of sensing layer, network layer, middleware layer and applications layer which are to manage data effectively and intelligently is the core research challenge in IoT development [9]. In this paper, a similar four layered framework is proposed for an IoT based smart environment.

Many European and American countries and multinational companies are involved in designing and developing IoT based smart systems for powerful automated services. The new concept 'DIGITAL INDIA' proposed by Government of India has gained its momentum and because of which IoT is hot research area increasing popularity for industry, 
government and academia as well. This paper is focused to design and establish a smart environment integrating various sectors will have its significant contribution towards Digital India scheme. The paper is organized in the following manner. Section II depicts the architecture for integrated smart environment. Section III presents the functioning of IoT based information Kendra supported by cloud data and

services centre and administrative management centre.

Section IV emphasizes the challenges and issues in the proposed work. Section V concludes the paper with its future enhancement.

\section{ARCHITECTURE FOR INTEGRATED IoT BASED SMART ENVIRONMENT}

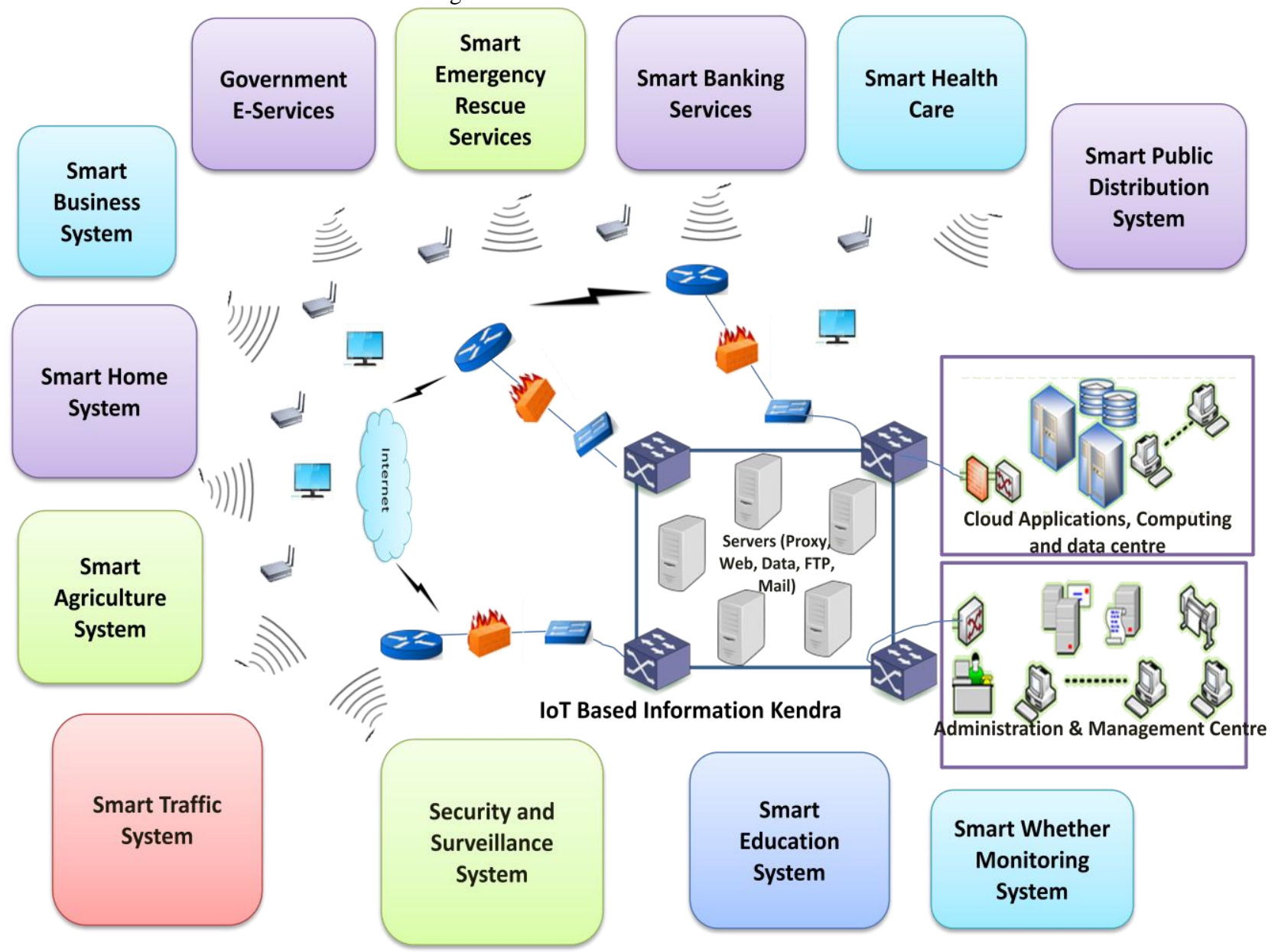

Figure 1 : Architecture for Integrated IoT based Smart Environment

This part of the paper explains the overall architecture for the smart environment. A layered framework for IoT based smart environment is also discussed. The functioning of RFID system is explicated. The various possible business applications and sectors to form the smart environment as well are dealt.

\subsection{Integrated Smart Environment}

The integrated smart environment is designed with the various business applications like smart home system, smart health care, smart traffic surveillance, smart business system, smart agriculture system, smart E-governance system, smart whether monitoring system, smart education system, smart rescue and emergency system, Smart public distribution system and smart banking system. RFID tags and sensors are connected to the objects and things which are in the various business applications of the smart environment. The RFID system comprises of RFID antenna and readers infer the information from various domains and send them to middleware for further processing of data to be sent to IoT based information Kendra through conventional network. In IoT based information Kendra, data is processed based on the application where the actual data is inferred. The IoT information Kendra is supported with cloud applications and data centre and management administration centre for recommended external services and billing related services respectively.

\subsection{Layered Framework for IoT based smart environment}

The layered framework for IoT based smart environment consists of four layers as shown in figure 2 . They are device layer (perception layer), middleware layer, network layer and application layer. Each layer is described below in short.

\subsubsection{Perception layer}

Perception layer is also known as device layer. All the sensor devices, RFID tags and various other physical objects belong to this layer. This layer is supported by the sensor network consists of sensors, RFID antennas, RFID readers. The sensor devices and RFID tags in the layer help to collect specific information related to the objects in the different business domains. The collected information may be location, temperature, sound, pressure, heart pulse, humidity, climatic 
condition and so on. The inferred data is sent further to middleware layer.

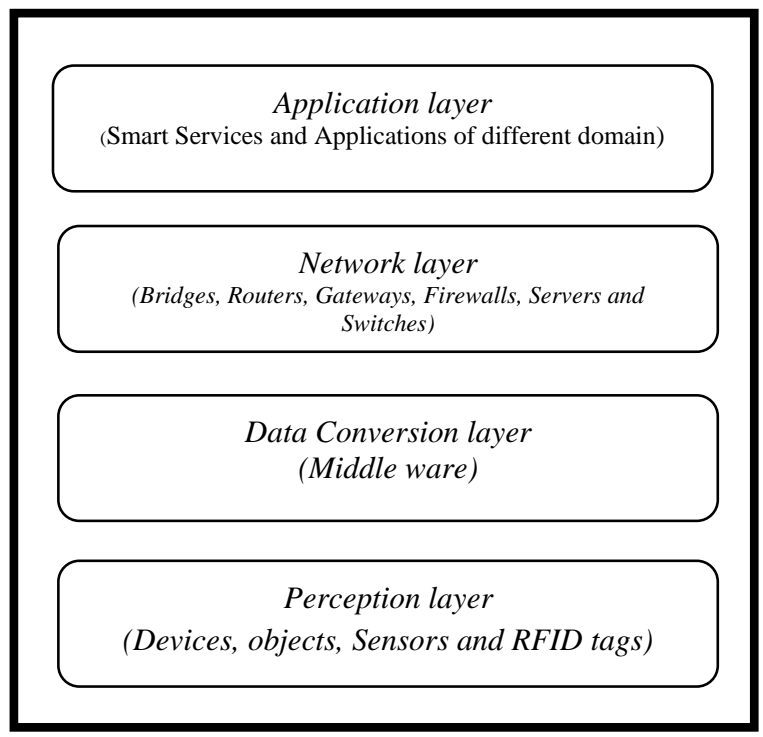

Figure 2: Layered Framework for IoT based

\subsubsection{Data Conversion Layer}

There may be several hetrogeneous signals from sensors and RFID tags in the tiny devices and objects attached to business applications integrated in the smart environment. The data collected in the form of hetrogenous signals can not be sent to the conventional network for further processing of data. Majority of the signals may be in analogous form. So, this layer is responsible for converting different signals inferred into data that could be sent through the conventional network. This layer consists of middleware which supports the conversion task.

\subsubsection{Network Layer}

Network Layer is also known as transport layer which is responsible for fetching the data to IoT information kendra for processing. The decision making process based the on the data sent will be supported with cloud applications data centre and an adminstrative management centre. This layer is functions based on TCP/IP as it lays on conventional network. It consists of gateways, routers, switches, firewalls and servers.

\subsubsection{Application Layer}

The application layer is comprised of all the business applications in the smart environment proposed. The functioning of each business sector differs by their applications and services. This layer is responsible for the decision making process based the data inferred from the objects and devices attached to the business domains.

\subsection{RFID System}

RFID system contains the sensors and RFID tags attached tothe devices and objects in the business applications. The signals from the sensors and RFID tags received by the RFID antena. The RFID reader perceives the signals and in turn sends them to the middleware. The middleware onverts the signals into readable data by digital system. The data converted sent to IoT based information kendra

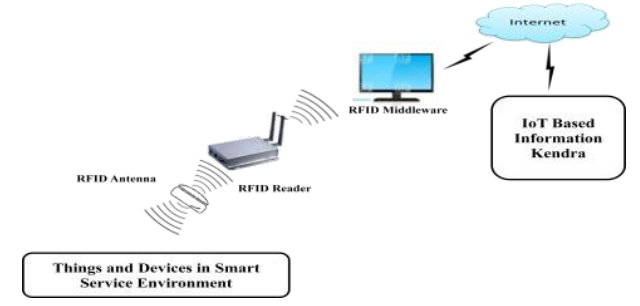

Figure 3. RFID System

\subsection{Feasible Applications}

\subsection{1 . Smart Home}

IoT enables designing a smart home by variety of tasks like controlling appliances, power and gas consumption, home security, emergency identification and other similar applications

\subsubsection{Smart Educational Environment:}

Student and Staff attendance, Class room and exam hall surveillance, Library book tracking, Laboratory equipments identification, campus security and emergency alert are few applications which may devices part of smart educational environment.

\subsubsection{Smart Health Care:}

IoT will facilitate life saving applications in the health care domain. Applications like patient health monitoring, doctors, workers and patient identification are few applications of this sector.

\subsubsection{Smart Security and Emergency:}

Tracking of people, places and movable and immovable assets, Identification of strange things and people, emergency alert by alarming are some the applications employed in security and emergency domain.

\subsubsection{Smart Agriculture and Whether Monitoring:} variety of sensors in the agriculture form will sense data like water consumption level, animals alerting service, soil condition based on fertilizers, crop status monitoring are some the applications devised in agriculture domain.

\subsubsection{Smart Traffic Surveillance:}

IoT supported by sensors and RFID tags can track the vehicles. Identification of vehicles violating traffic regulations, accident and emergency identification and alert, road condition alert, speed limit alert, lane control, parking identification and no parking alert are few applications may be

\subsubsection{Smart Predictor of Disasters and}

\section{Emergency:}

The sensors with the help of simulators will alert disasters like fire and other natural disasters like land slide, earth quake and flood. Emergency alert, Ambulance Alert, SoS alert are some of the feasible applications.

\subsubsection{Smart Business System:}

Sensors and the RFID tags fixed the business environment will ease its functioning smarter. Inventory control, goods and commodities identification, purchase or sales billing, surveillance in the business concern, Customer identification, Business places like shops, malls, hotels identifications alert are few applications in the business domain. 


\subsubsection{Smart Banking System:}

Banking is a big domain has numerous stack holders. Multiple applications can be employed to make the banking sector smarter than existing. Customer identification, Transactions alert, Security alert, E-services, ATM locating, Smart Debit and Credit Cards embedded with RFID tags, Smart cheque and demand draft services, RFID enabled business cards to be used at PoS are the applications introduced to the banking sector using IoT.

\subsubsection{Smart Public Distribution System:}

The regulation of public distribution system may be possible with the help of IoT environment. RFID embedded Ration Card, Customer identification, stock alert, security surveillance, customers' alert for the products distribution, smart billing, quality check measures are some applications can be thought of in PDS.

\subsubsection{Government E-Services:}

Aadhar cards can be converted to into cards entrenched with RFID tags. This will enable the government E-services much smarter than today. People identification and locating, RFID tags enabled Electronic Voter ID cards, Government Services and employment alerts, Government Order notifications, Public notice alert to the needy, Government Offices locating, Government programmes alerts are some the applications may be devised in the proposed smart environment.

\section{IOT BASED INFORMATION KENDRA}

The proposed smart environment is supported with IoT based Information Kendra which processes the information inferred from the various business applications using RFID and sensor networks. The architecture is constructed with the backbone of internet using TCP/IP. There are specific servers namely Web server, Proxy server, Mail Server, Data server, FTP server which connected with IP core switch from which the data packets are directed to the intended destiny via firewall and through router which is the gateway for the IoT information centre. Data server holds the collected and processed data to be fetched for the required services. Mail server is used to facilitate mail alerts and relevant services to the smart environment. Web server possesses web based data to be supplied as web contents of the web sites of the various business sectors in the smart environment. Proxy server facilitates the network connections in the proposed smart environment. FTP server is responsible transfer of files using HTTP in the IoT based information Kendra. When the different clients of various business domains in the smart environment need the information, IoT based Information Kendra will provide the data or services with the assistance of cloud applications and data centre.

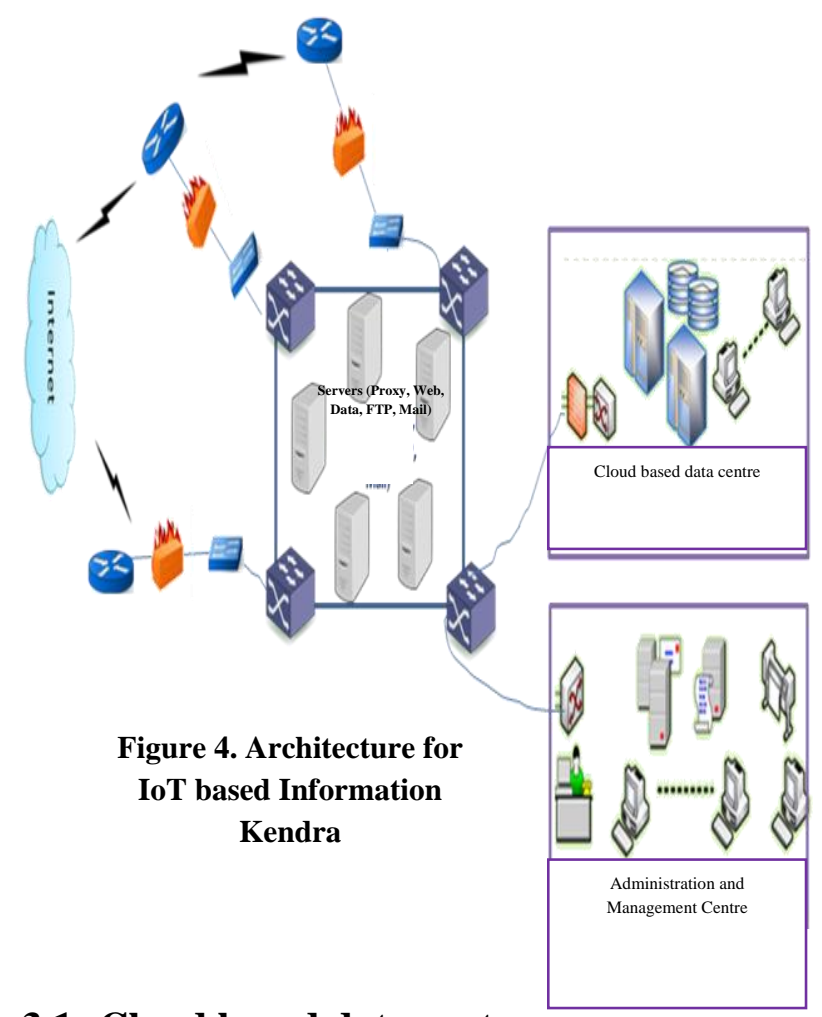

\subsection{Cloud based data centre}

The IoT based Information Kendra is attached with Cloud based computing data centre which comprises of cloud servers and data processing applications that help IoT based Information Kendra with relevant services required by the business applications in the smart environment. Each business domain is need of variety of services like security, emergency and whether alerts, automated tasks, mail and mobile message alerts, information dissemination, home appliances control. These services are rendered based on the data collected from the different business applications using RFID and sensor networks.

\subsection{Administration and Management Centre}

Another unit attached to IoT based Information Kendra is Administration and Management Centre which is responsible for the managerial operations of IoT based Information Centre. Some services may be provided free of cost and the rest of the services may be charged according to the expenses met. Categorization of services, billing, quality assurance, maintenance of sensors and equipments at the smart environment and other managerial and administrative functions are the responsibilities of this centre.

This IoT based information Kendra can be established to cover the geographical area of a district or a mandal. All the possible business applications proposed may create smart environment which may be affixed to the IoT Information Kendra for the data processing and other services required.

\section{CHALLENGES AND ISSUES}

The proposed IoT based smart environment will offer enormous benefits to the society. Many business sectors and government services are integrated through this smart environment. Several devices and objects are connected with the help of RFID and sensors. Thus, this proposed smart environment is a multifaceted in nature. Hence, challenges 
and issues are many to be addressed. Some of the issues and challenges discussed below.

\subsection{Object Naming}

The proposed smart environment will connect several thousands of devices and objects for different services. Every device and object needs to be uniquely identified over the network. So, a dynamic mechanism of object naming and identification is needed to manage large number of devices connected.

\subsection{Data Conversion}

The signals and data inferred from the connected devices and objects will vary in their nature and hence, they cannot be transmitted via conventional network using internet. Effective methods of data conversion to be used for making the data compatible for further processing by IoT based Information Kendra.

\subsection{Data Conversion}

Many applications from various domains will have different identification technologies for the devices and objects. Several clients will be involved accessing and making use of the services by this smart environment. It is essential to take necessary steps to take proper privacy measures and prevent unauthorized access of the devices and objects. There is another possibility where people may not be aware of the sensors fixed, so, it is good to regulate the privacy of human being as well.

\subsection{Interoperability}

The devices and objects are heterogeneous in their functioning. Each device and object will use their own technologies and they may not be compatible to use the services of others. Interoperability to all the objects and devices like RFID tags, sensors should be ensured. The manufacturing of devices and objects are not with same standard and the standardization object and device manufacturing is needed

\subsection{Quality of Service}

As several millions of data to be transferred for various services, there may be lack of quality of services. It is necessary to take steps to ensure the quality measure to provide better services to different applications in the smart environment.

\subsection{Security Attacks}

Information from the devices and objects connected to this smart environment are prone to security attacks like firsthand attack, gossip attack, observation attack, inference attack, automated invasion attack [1]. A proper security mechanism should be devised to address these mentioned attacks.

\subsection{Data encryption and key management:}

Data encryption is also a major concern in the proposed smart environment. Encryption algorithms like AES, RSA, DH use keys of longer in length whereas Elliptic Curve Cryptography algorithm uses shorter length key. ECC is recommended for the data encryption because the devices and objects are very tiny and the heavy weight key exchange will prevent effective functioning [10].

\subsection{Security for Hardware}

The IoT Smart Environment will cover larger geographical area. There is a chance of intruders' interventions towards the objects and sensor devices. Sensor data may be inferred by authorized sources by setting up their own RFID readers and other devices. Thus it is necessary to protect the devices and objects attached in the smart environment from intruder's access, physical damage and malfunctioning.

\subsection{Network Congestion}

As millions of objects and devices connected, certainly there will be network congestion in data transmission. The future research on IoT should also focus to avoid network congestion without data loss. Security measures should be taken to ensure the transmission of data without the external interferences.

\section{CONCLUSION}

The proposed architecture for the IoT based Smart Environment will be another phase lift in Next Generation Network. A revolution in the domestic appliances is possible with the help of technological advancements. This paper described how IoT could integrate the different business domain under smart environment duly supported by IoT Information Kendra. The IoT information Kendra will serve general public with many services if they are established in each district or region. This paper has brought out some feasible applications in each domain. As the IoT based smart environment has enormous benefits the number of challenges and issues are many and they have to be addressed properly. The deployment of this proposed IoT based Smart Environment will be difficult but at the same time it has numerous benefits to the society in near future.

\subsection{Future Enhancements}

The future research in IoT may concentrate on the challenges and issues discussed in the paper. Security is a major concern in the proposed environment integrating the different business applications. Security architecture for the proposed work may be designed in future to give integrated solutions solving different security issues like key management, intruder's attacks, unauthorized access and network congestion. Future research efforts in IoT should also be focused to resolve interoperability as many devices and objects with heterogeneous functionalities are attached to the smart environment. The possible services with different nature with same data inferred and when required by many may cause poor quality of service. Research endeavors in future should also deal with the quality of service.

\section{REFERENCES}

[1] Dieter Uckelmann, Mark Harrison, Florian Michahelles, 2011. An Architectural Approach Towards the Future Internet of Things. Architecting Internet of Things by Springer, 1-22.

[2] Kevin C. Desouza, David Swindell, Kendra L. Smith, Alison Sutherland, Kena Fedorschak, and Carolina Coronel, 2015. Issues in Technologies innovation, (May 2015)

[3] Jayavardhana Gubbi a , Rajkumar Buyya b, Slaven Marusic a , Marimuthu Palaniswami, 2013. Internet of Things (IoT): A vision, architectural elements, and future directions. Future Generation Computer Systems 29, $1645-1660$

[4] Elkhodr, M. Shahrestani, S, Hon Cheung, 2013. The Internet of Things: Vision \& Challenges. TENCON IEEE Spring Conference, 218-222.

[5] K. Ashton, 2009. That 'Internet of Things' Thing. RFid Journal, 97-114.

[6] Jebah Jaykumar, Abishline Blessy, 2014. Secure Smart 
Environment Using IoT based on RFID. International Journal of Computer Science and Information Technologies, Vol. 5 (2), 2493-2496.

[7] Manik Lal Das, 2013. Strong Security and Privacy of RFID System for Internet of Things Infrastructure. Springer, Security, Privacy, and Applied Cryptography Engineering, 56-69.

[8] TD Division, TRAI, 2015. Internet of Things. Technology Digest, Bulleting of Telecom Technology, Issue 23 July 2015.
[9] Meng Ma, Ping Wang, Chao-Hsien Chu, 2013. Data Management for Internet of Things: Challenges, Approaches and Opportunites. IEEE International Conference on Green Computing and Communications and IEEE Internet of Things and IEEE Cyber Physical and Social Computing, $1144-1151$

[10] Hui Suo, Jiafu Wan, Caifeng Zou, Jianqi Liu, 2012. Secruity in the Internet of Things: A Review. International Conference on Computer Science and Electronic Engineering, IEEE Computer Society, 648-651. 